\title{
Phosphorylation of EBP50 negatively regulates $\beta$-PIX-dependent Rac1 activity in anoikis
}

\author{
This article has been corrected since Advance Online Publication and a Erratum is also printed in this issue
}

\author{
J-Y Chen ${ }^{1}$, Y-Y Lin ${ }^{1}$ and T-S Jou ${ }^{*, 1,2,3}$
}

We demonstrated a protein kinase C (PKC)-dependent phosphorylation of canine ezrin/radixin/moesin (ERM)-binding phosphoprotein 50 (EBP50) at serine $347 / 348$ by site-directed mutagenesis and a phospho-specific antibody. Cell fractionation and confocal imaging revealed the relocation of EBP50 from the plasma membrane to cytosol that accompanied this phosphorylation event. Increased phosphorylation at these serine residues led to the dissociation of EBP50 from ezrin and $\beta$-PIX, which are two upstream regulators of Rac1 activation. Cells overexpressing an EBP50 mutant, mimicking serine $347 / 348$ phosphorylation, became refractory to hepatocyte growth factor-induced cell spreading and scattering, which is normally mediated by Rac1 activation. Detachment of cells from the substratum also elicited an increase in EBP50 phosphorylation, apparently due to counteracting activities of PKC and protein phosphastase $2 \mathrm{~A}$, which resulted in decreased Rac1 activation and induction of anoikis. Cells overexpressing an EBP50 mutant defective in serine 347/348 phosphorylation did not undergo apoptosis in suspension culture. These studies reveal a signaling cascade in which different phosphorylation states and subcellular localization of EBP50 regulate Rac1 function.

Cell Death and Differentiation (2012) 19, 1027-1037; doi:10.1038/cdd.2012.4; published online 3 February 2012

Scaffold proteins have many modular domains that mediate protein-protein interactions involved in orchestrating different signaling cascades. As a result, scaffold proteins are thought to act as stable structural proteins that facilitate many cell signaling pathways. However, this traditional role of scaffold proteins has been challenged, ${ }^{1}$ and the dynamic role of scaffold as both an activator and suppressor of cellular signaling remains poorly characterized. Furthermore, the factors that modulate such a bifunctional role of scaffold proteins remain largely unknown.

Ezrin/radixin/moesin (ERM)-binding phosphoprotein 50 (EBP50) is a scaffold protein with two tandem PDZ domains and a C-terminal ERM-binding domain., ${ }^{2,3}$ EBP50 binds proteins that contain a PDZ-binding motif including ion exchangers, ${ }^{4,5}$ ion channels, ${ }^{6} \mathrm{G}$ protein-coupled receptors, ${ }^{7-9}$ growth factor tyrosine kinase receptors ${ }^{10,11}$ and PTEN. ${ }^{10}$ EBP50 participates in the modulation of ion transport, organization of apical microvilli, cancer development, and the trafficking and stabilization of membrane proteins. ${ }^{9,12-14}$ Understanding the mechanisms that regulate the function of EBP50 is, therefore, a critical problem.

Ras family proteins are locked in a GDP-bound, inactivated state by binding GDI in the GTP-rich cellular environment. ${ }^{15,16}$ Dissociation of small GTPases from GDI is required, but is not sufficient for their activation. GEFs drive the exchange of GTP for GDP, leading to the activation of small GTPases. ${ }^{17}$ EBP50 can activate Rho-family small GTPases by recruiting ezrin, which is thought to cause the dissociation of GDI from the small GTPase, and $\beta$-PIX, a Rac1 GEF that contains a PDZ-binding motif. ${ }^{18,19}$

EBP50 is a serine/threonine-phosphorylated protein. ${ }^{3}$ The phosphorylation state of EBP50 regulates the accessibility of its PDZ domains for cognate ligands and the formation of EBP50 oligomers, both of which are required for EBP50 to act as a scaffold protein. ${ }^{14,20}$ Several kinases phosphorylate EBP50. G protein-coupled receptor kinase 6A (GRK6A) constitutively phosphorylates rabbit EBP50 at serine 289, which may facilitate EBP50 oligomerization. ${ }^{21,22}$ During mitosis, rabbit EBP50 is phosphorylated by cyclin-dependent kinase Cdc2 at serine 279 and 301, which inhibits EBP50 oligomerization. ${ }^{23}$ In addition, protein kinase $\mathrm{C}$ (PKC) phosphorylates human EBP50 at serine 162,339 and 340 , which regulates the affinity between the PDZ domains of EBP50 and the PDZ ligands, and promotes EBP50 oligomerization. ${ }^{14,20,24,25}$ Recently, it has been known that microvilli assembly and the activity of cystic fibrosis transmembrane conductance regulator are modulated by EBP50 phosphorylation. ${ }^{14,25}$ Although the accumulating evidence supports the functional importance of EBP50 phosphorylation, the underlying regulatory mechanisms involved remain poorly understood.

We exploited the availability of an antibody that specifically recognizes dual serine phosphorylation in the C-terminal

\footnotetext{
${ }^{1}$ Graduate Institute of Molecular Medicine, National Taiwan University, No.7, Chung-Shan S. Road, Taipei 100, Taiwan; ${ }^{2}$ Department of Internal Medicine, National Taiwan, University Hospital and National Taiwan University College of Medicine, No.7, Chung-Shan S. Road, Taipei 100, Taiwan and ${ }^{3}$ Graduate Institute of Clinical Medicine, National Taiwan University College of Medicine, No.7, Chung-Shan S. Road, Taipei 100, Taiwan

${ }^{*}$ Corresponding author: T-S Jou, Graduate Institute of Molecular Medicine, National Taiwan University, No. 7, Chung-Shan S. Road, Taipei 100, Taiwan.

Tel: + 8862 23123456, ext. 67625; Fax: + 8862 23709820; E-mail: jouts@ ntu.edu.tw

Keywords: EBP50; Rac1; $\beta$-PIX; anoikis; PP2A

Abbreviations: EBP50, ERM binding phosphoprotein 50; ERM, ezrin/radixin/moesin; GDI, guanine nucleotide dissociation inhibitor; GEF, guanine nucleotide exchange factors; GST, glutathione S-transferase; HGF, hepatocyte growth factor; PDZ, postsynaptic density 95/discs large/zona occludens-1; PKC, protein kinase C; PMA, Phorbol-12-myristate-13-acetate; PP2A, protein phosphatase 2A

Received 17.8.11; revised 20.12.11; accepted 05.1.12; Edited by RA Knight; published online 03.2.12
} 
ezrin-binding domain of EBP50, and uncovered a counteracting PKC/protein phosphastase 2A (PP2A) phosphatase activity on EBP50 upon cellular deprivation of substratum interaction. We propose that enhanced phosphorylation of EBP50 disrupts an EBP50-ezrin- $\beta$-PIX complex, which leads to downregulated Rac1 activity, and compromises the ability of cells to resist anoikis.

\section{Results}

Phosphorylation of serine 347 and 348 induces the subcellular redistribution of EBP50. To investigate the cellular effects of EBP50 phosphorylation at serine 347/348, a series of canine EBP50 mutants were constructed (Figure 1a) and transiently expressed in polarized Madin Darby canine kidney (MDCK) cells. Although the phosphorylation-defective mutant (FE50_AA) and the mutants mimicking single phosphorylation at either serine 347 or 348 (FE50_DA, FE50_AD) were all localized to the subapical region of MDCK cells, similar to the distribution of wild-type EBP50 (FE50_WT) (Figure 1b), the mutant mimicking dual phosphorylation at serine 347/348 (FE50_DD) was also strongly localized in the cytosol.
PKC phosphorylates human EBP50 at serine 339 and 340 in vitro, which corresponds to serine 347 and 348 in canine EBP50 (Supplementary Figure 1). To characterize the effect of PKC on EBP50 distribution in vivo, we treated MDCK cells overexpressing FE50_WT with phorbol-12-myristate-13acetate (PMA) to activate PKC. Upon PKC activation, FE50_WT (Figure 1c, upper panel) and endogenous EBP50 (Figure 1d) had subcellular distributions similar to the phosphorylation-mimicking mutant FE50_DD, and FE50_WT was recovered in the cytosolic fraction following cell fractionation (Figure 1c, bottom panel). However, PMA did not affect the subcellular distribution of FE50_AA (Figure 1c), confirming that the phosphorylation state of serine 347 and 348 determines the subcellular localization of EBP50.

EBP50 is phosphorylated at both serine 347 and 348 in vivo. Although PKC-dependent EBP50 phosphorylation of serine 347 and 348 had been demonstrated with recombinant protein $^{20}$ and an EBP50 mutant with a C-terminal truncation, ${ }^{24}$ the biological regulation and consequence of this phosphorylation event are largely unknown. To further investigate EBP50 phosphorylation at serine 347/348, we

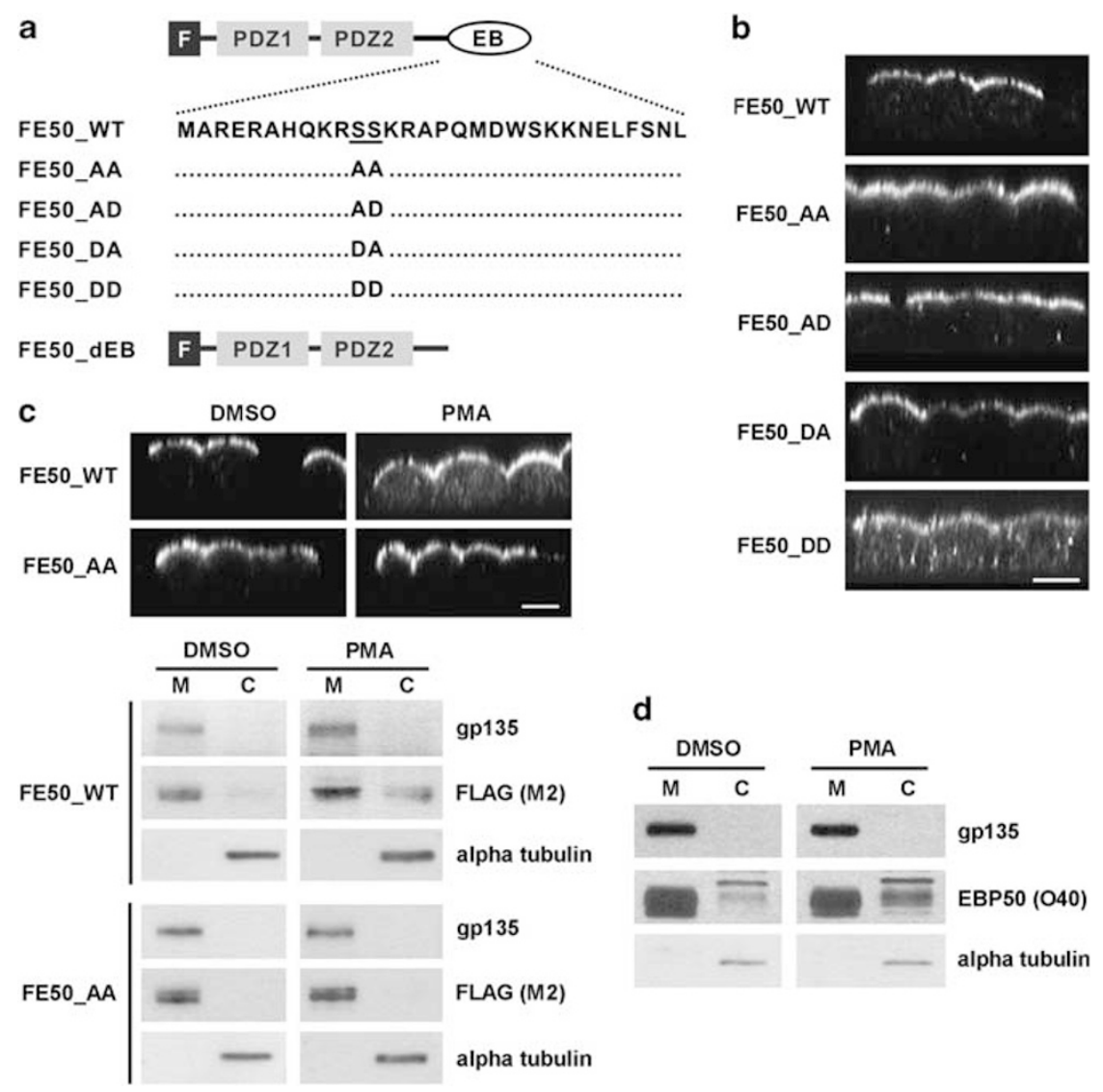

Figure 1 Phosphorylation of both serine 347 and 348 induces cytosolic redistribution of apical cortical protein EBP50 in polarized MDCK cells. (a) Schematic diagram of EBP50 constructs. Amino-terminal FLAG-tagged (F) wild-type canine EBP50 (FE50_WT) and various mutants in which serine residues at position 347 and 348 (underlined) in the ezrin-binding region (EB) were replaced with either alanine (A) or aspartate (D) as indicated. FE50_dEB, a FLAG-tagged mutant lacking EB. PDZ, PDZ domain. (b) MDCK cells transiently expressing the indicated EBP50 constructs were cultured on Transwell filters for 2 days and processed for immunofluorescence using rabbit anti-FLAG antibody. (c) Polarized MDCK cells expressing FLAG-tagged wild type or phosphorylation defective EBP50 mutant were serum-starved overnight before being treated with $30 \mathrm{nM} \mathrm{PMA}$ or DMSO for $30 \mathrm{~min}$. Then, cells were processed for immunofluorescence (upper panel) or fractionated into membrane (M) or cytosolic (C) fractions as described in 'Materials and methods' before being processed for western blotting (lower panel). GP135 and alpha tubulin were used as plasma membrane and cytosolic markers, respectively. (d) MDCK cells were treated with PMA or DMSO and fractionated as described above. All X-Z sections were acquired by confocal microscope. Bar, $10 \mu \mathrm{m}$ 
generated an antibody (pEBP50(Ser347-8)) that specifically recognized EBP50 phosphorylated at both Ser347 and 348. This antibody recognized the dually phosphorylated antigenic peptide, but neither the singly phosphorylated nor the unphosphorylated control peptide was recognized (Figure 2a). The antibody also detected a robust signal of FE50_WT from immunoprecipitated cell lysate after PMA treatment (Figure $2 b$ ). In contrast, the phosphorylationdefective mutant FE50_AA was barely detected either before or after PMA treatment (Figure $2 b$ ).

We examined the time course of phosphorylation of endogenous EBP50 following PKC activity with PMA. At 15 min after PMA treatment, pEBP50(Ser347-8) recognized an intense signal of slower electrophoretic mobility, which was sustained for $45 \mathrm{~min}$ (Figure 2c). As the slower electrophoretic mobility signal decreased, a faster electrophoretic mobility signal was detected, which persisted for a longer time period. EBP50 knockdown resulted in the loss of the slower but not the faster electrophoretic mobility signal. Therefore, the faster electrophoretic mobility signal is likely a nonspecific background signal (Supplementary Figure 2). Consistent with results from the imaging studies using phosphorylation mutants (Figures $1 \mathrm{~b}$ and $\mathrm{c}$ ), cell fractionation demonstrated that as endogenous EBP50 became increasingly cytosolic, PKC-dependent phosphorylation of serine 347 and 348 increased (Figure 2d). Taken together, these results verified the existence of endogenous EBP50 phosphorylated at Ser347/348 in vivo, which had been suggested to be PKC-dependent. ${ }^{20,24}$

Phosphorylation of EBP50 at Serine 347/348 modulates its interaction with ezrin. Ezrin-radixin-moesin family proteins have been suggested to retain EBP50 at the apical membrane of polarized epithelial cells. ${ }^{26,27}$ FE50_dEB, an EBP50 mutant lacking the ezrin-binding domain, was localized exclusively in cytosol (Figure 3c), similar to the a

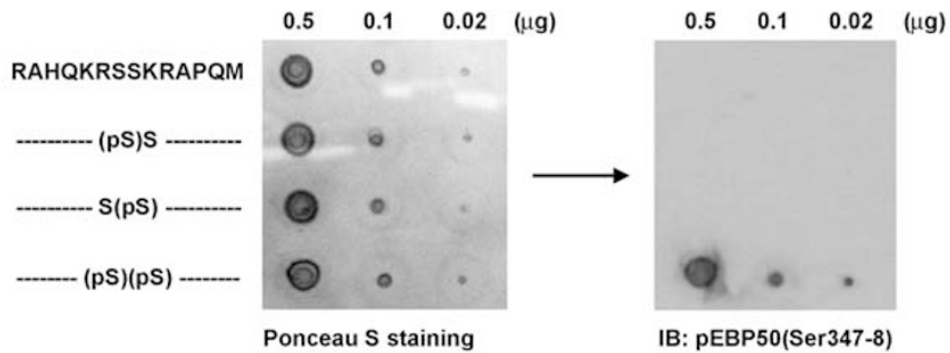

b

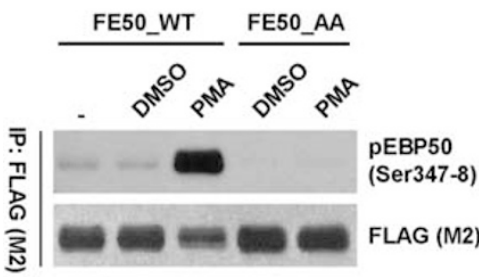

C

\begin{tabular}{llllll}
\multicolumn{5}{c}{ PMA (hr) } \\
\hline 0 & $1 / 4$ & $1 / 2$ & 1 & 2 & 3
\end{tabular}

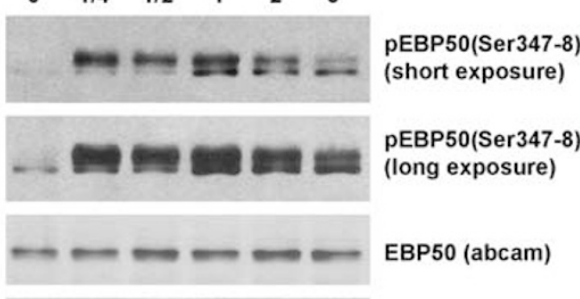

-- alpha tubulin

d

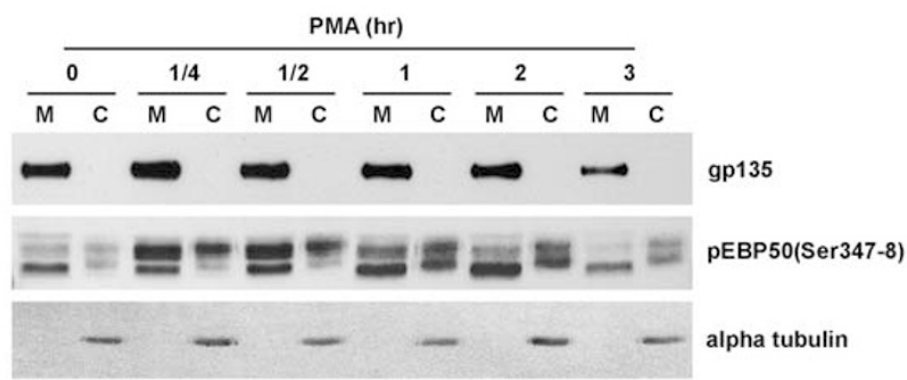

Figure 2 PMA induces phosphorylation of EBP50 at both serine 347 and 348 in vivo. (a) The polyclonal antibody against EBP50 phosphorylated at both serine 347 and 348 , pEBP50(Ser347-8), was generated and purified as described in 'Materials and methods'. The antigenic peptide, which was phosphorylated at serine 347 and 348 (pS)(pS), and other control peptides were dot blotted onto nitrocellulose membrane and then tested by western blotting analysis using the pEBP50(Ser347-8) antibody. (b) MDCK cells expressing FE50_WT and FE50_AA were treated with DMSO or PMA (30 nM, $30 \mathrm{~min})$. Cell lysates were extracted, immunoprecipitated with anti-FLAG M2 affinity resin, and processed for western blotting analysis using the pEBP50(Ser347-8). (c) MDCK cells were serum-starved overnight and then treated with $30 \mathrm{nM}$ PMA for the indicated times. Cell lysates were resolved by SDS-PAGE and analyzed by western blotting using pEBP50(Ser347-8) and a commercial antibody to detect total EBP50. The alpha tubulin blot indicates equal loading of samples. (d) PMA-induced cytosolic redistribution of EBP50 is accompanied by phosphorylation at serine 347 and 348 . MDCK cells were serumstarved and treated with PMA $(30 \mathrm{nM})$ for the indicated times and then fractionated into membranes $(\mathrm{M})$ or cytosol $(C)$. The fractionated lysates were then resolved by SDS-PAGE and analyzed by western blotting using pEBP50(Ser347-8). GP135 and alpha tubulin were used as the plasma membrane and cytosolic marker, respectively 
a

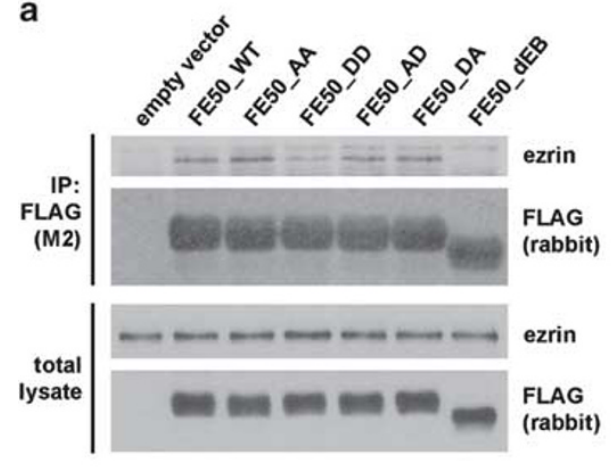

b

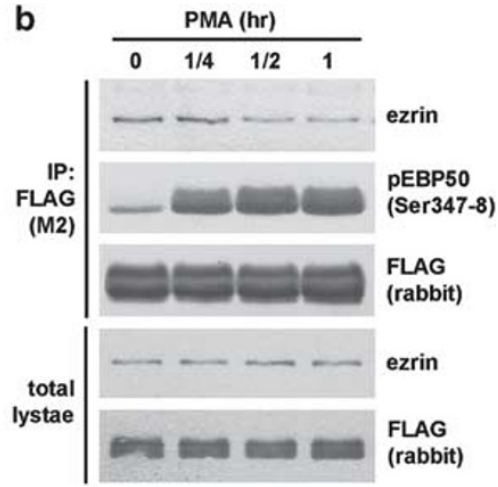

c

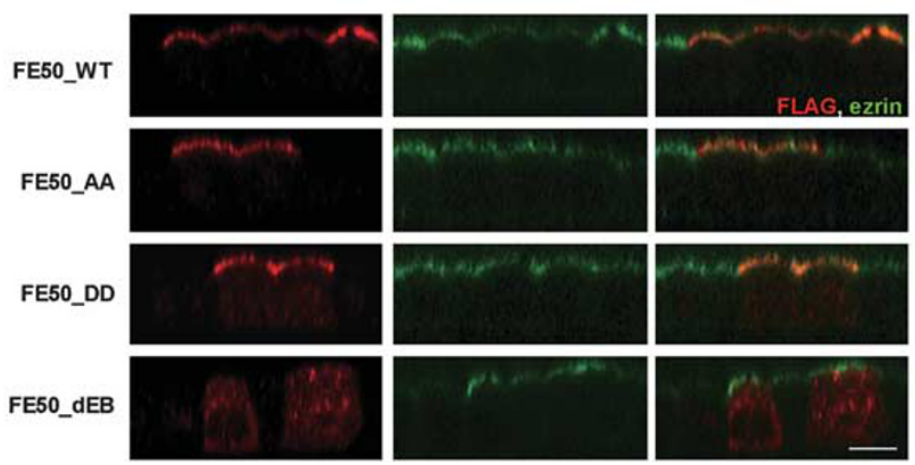

Figure 3 Phosphorylation of EBP50 serine 347 and 348 dissociates EBP50 from ezrin. (a) Cell lysates from HEK293 cells transiently expressing the indicated EBP50 constructs were immunoprecipitated with mouse anti-FLAG antibody (M2). Then, the precipitated samples were analyzed by western blotting using ezrin or rabbit anti-FLAG antibody. (b) MDCK cells stably expressed FE50_WT and were serum-starved overnight, and subsequently treated with $30 \mathrm{nM}$ PMA for the indicated times. Then the cell lysates were immunoprecipitated using anti-FLAG M2 affinity resin. The precipitated samples were analyzed by western blotting with anti-ezrin, rabbit anti-FLAG or pEBP50(Ser347-8) antibody. (c) MDCK cells transiently expressing the indicated EBP50 constructs were cultured at an instant confluent density on Transwell filters for 2 days and then processed for double immunofluorescence using antibodies against ezrin (green) and FLAG epitope (red), and examined by confocal microscopy. Bar, $10 \mu \mathrm{m}$

distribution of the mutant mimicking dual phosphorylation (FE50_DD) (Figure 1b).

We hypothesized that EBP50 phosphorylation at serine $347 / 348$ affected the interaction between EBP50 and ezrin. Although a similar amount of ezrin was co-immunoprecipitated with FE50_WT, FE50_AA, FE50_AD or FE50_DA, much less ezrin was co-immunoprecipitated with comparable levels of FE50_DD and the ezrin-binding defective mutant FE50_dEB (Figure 3a). In addition, less ezrin was co-immunoprecipitated with EBP50 upon PMA-induced PKC activation and phosphorylation of serine 347 and 348 (Figure $3 b$ ). Taken together, these results indicate that the phosphorylation of EBP50 at serine 347 and 348 attenuates the interaction between EBP50 and ezrin, and leads to EBP50 redistribution from the apical membrane to the cytosol. Although phosphorylation of serine 347 and 348 greatly diminished the interaction between EBP50 and ezrin, the apical distribution of ezrin was not affected by overexpressing either the FE50_DD or the FE50_dEB (Figure 3c), indicating that ezrin localization to the apical membrane was not dependent on the membrane localization of EBP50.

Serine 347 and 348 phosphorylation of EBP50 attenuates Rac1 activation in vivo. The formation of a complex of podocalyxin-EBP50-ezrin facilitates the activation of Rho-family GTPases. ${ }^{18}$ Previously, we identified a novel interaction between EBP50 and $\beta$-PIX, a specific GEF for
Rac1 and Cdc42, and proposed that the podocalyxinEBP50-ezrin complex recruited $\beta$-PIX and, thereby, enhanced the metastatic potential of renal cell carcinoma. ${ }^{19}$ To confirm the role of EBP50 in Rac1 activation in living cells, we measured Rac1 activity in EBP50 knockdown MDCK cells in the presence of MRC-5 conditioned medium, which induces Rac1 activation via bioactive hepatocyte growth factor (HGF). ${ }^{28}$ EBP50 knockdown not only resulted in diminished basal Rac1 activity but also significantly reduced the level of Rac1 activation compared with mock cells exposed to MRC-5 conditioned medium (Figure 4a). To exclude the possibility of artificial effect from stable clone selection, the same results were observed not only in two independent clones whose EBP50 expression had been successfully knocked down (Supplementary Figure 3a) but also in cells whose EBP50 expression was suppressed transiently using small-interfering RNA (siRNA) oligonucleotides (Supplementary Figure 3b).

As described above, EBP50 phosphorylation at serine $347 / 348$ reduced the interaction between EBP50 and ezrin, and caused the subcellular redistribution of EBP50 to the cytosol. Both events could disturb the formation of the podocalyxin-EBP50-ezrin complex and lead to the impaired Rac1 activation mediated by EBP50. We tested the effects of EBP50 phosphorylation at serine 347/348 on Rac1 activation by expressing siRNA-resistant FE50_reWT, FE50_reAA and FE50_reDD in the EBP50 knockdown cell line (siE50_16 in Supplementary Figure 3a). Compared with cells expressing 


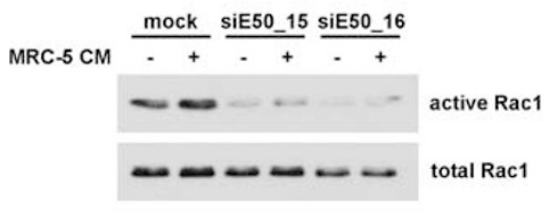

b

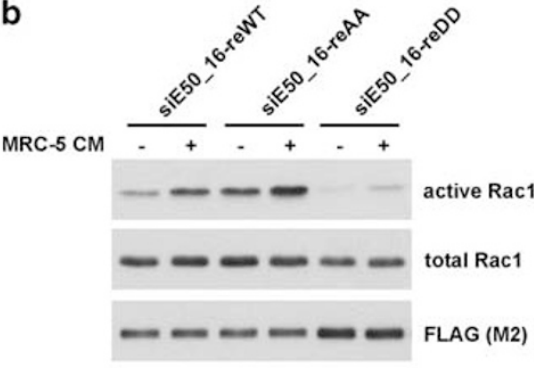

SIE50_16-reWT
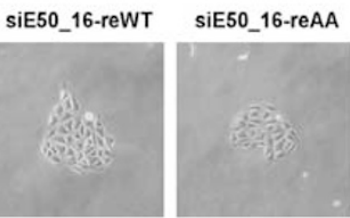

SiE50_16-reDD
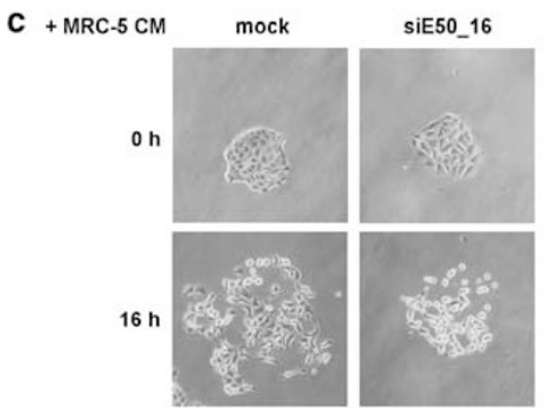
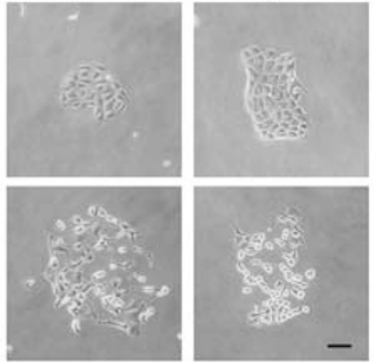

Figure 4 EBP50 serine 347 and 348 phosphorylation negatively regulates EBP50-dependent Rac1 activation. (a) The Rac1 activation assay was performed with MDCK mock-transfectant and two independent knockdown cell lines expressing siRNA targeted against endogenous EBP50 (siE50_15 and 16) before and after incubation with conditioned medium (CM) from MRC-5 fibroblasts. (b) Rac1 activation assay was performed in revertant MDCK clones stably expressing siRNA-resistant FLAG-tagged wildtype EBP50 (siE50_16-reWT), alanine-alanine mutant (siE50_16-reAA) and aspartate-aspartate mutant at the 347 and 348 amino-acid positions (siE50_16-reDD). The data shown here are representative results from three independent experiments. (c) MDCK stable cell lines expressing the indicated EBP50 constructs were imaged before and $16 \mathrm{~h}$ after being treated with MRC-5 CM to document cell scattering. Bar, $40 \mu \mathrm{m}$. MDCK cells stably transfected with the empty vector (pSilencer 1.0) were denoted as mock transfectant

FE50_reWT or FE50_reAA, Rac1 activity was significantly lower in cells expressing FE50_reDD under either basal conditions or after treatment with MRC-5 conditioned medium (Figure 4b). Note that at least two independent revertants with comparable EBP50 expression levels (Supplementary Figure 4a) were analyzed to exclude clonal selection of these effects. In addition, there was no difference in the levels of Rac1 activation among individual clones reexpressing the indicated constructs, and only the result representing one clone for each construct are shown for brevity (Figure 4b). Rac1 activity was also measured in additional stable cell lines (Supplementary Figure 4b) derived from an independent EBP50 knockdown cell line (siE50_15 in Supplementary Figure 3a) reexpressing the siRNA-resistant constructs FE50_reWT, FE50_reAA and FE50_reDD with similar results (Supplementary Figure 4c). In addition, FE50_reDD-expressing cells were defective in HGFinduced cell scattering, a process requiring Rac1 activity. ${ }^{29}$ The FE50_reDD-expressing and EBP50-knockdown cells maintained an adhesive phenotype, whereas mock transfectant, FE50_reWT- and FE50_reAA-expressing cells, all responded to MRC5 fibroblast conditioned medium (Figure 4c) by increasing cell spreading on the substratum.

EBP50 phosphorylation at serine $347 / 348$ modulates its interaction with $\beta$-PIX. $\beta$-PIX (also known as ARHGEF7) is a RhoGEF involved in various small GTPase signaling pathways. $^{30} \beta$-PIX contains a PDZ-binding motif at its C-terminal end. We recently demonstrated that EBP50 interacts with $\beta$-PIX through its first PDZ domain, and $\beta$-PIX associates with the podocalyxin-EBP50-ezrin complex. ${ }^{19}$ The interaction between EBP50 and $\beta$-PIX was confirmed by co-immunoprecipitation (Figure 5a). Both basal and MRC-5 conditioned medium-dependent Rac1 activation decreased upon $\beta$-PIX knockdown, confirming the involvement of $\beta$-PIX in the Rac1 activation, using MRC-5 conditioned medium (Figure 5b). Because the accessibility of EBP50 PDZ domains for binding partners can be modulated by phosphorylation at the ezrin-binding domain of EBP50, ${ }^{14,20}$ we hypothesized that phosphorylation of ser347 and 348 residues in EBP50 might affect the binding between EBP50 and $\beta$-PIX. Using glutathione S-transferase (GST) fusion protein pull-downs, we found that the C-terminal of $\beta$-PIX interacts with ezrin only in the presence of EBP50 and not in the EBP50 knockdown cells (Figure $5 \mathrm{c}$ ). This result indicates that $\beta$-PIX binds to ezrin indirectly through EBP50. Significantly, a mutant $\beta$-PIX lacking the PDZ motif did not bind to EBP50 or ezrin (Figure $5 \mathrm{c}$ ), confirming that $\beta$-PIX interacts with EBP50 through its C-terminal PDZ motif.

Although the reexpressed FE50_reDD protein was more in the initial loading lysate than that of FE50_reAA, there was much less pulled-down EBP50 and ezrin in cells expressing FE50_reDD than those expressing FE50_reAA (Figure 5c, left panel). This result, together with the fact that PMA treatment reduced the interaction between wild-type EBP50 and $\beta$-PIX (Figure $5 \mathrm{c}$, right panel), indicates that PKCdependent EBP50 phosphorylation at these critical serines directly affects the EBP50-ezrin- $\beta$-PIX complex.

The effect of amino-acid substitutions at serine 347/348 on the interaction between EBP50 and $\beta$-PIX was further verified by pull-downs from lysates of human embryonic kidney (HEK) 293 cells transiently expressing phospho-resistant and phosphoserine-mimicking EBP50 mutants. Although substitution 

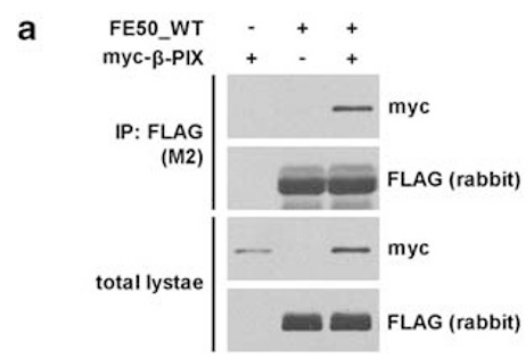

C

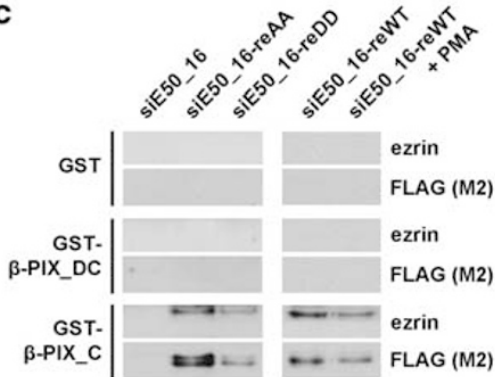

b

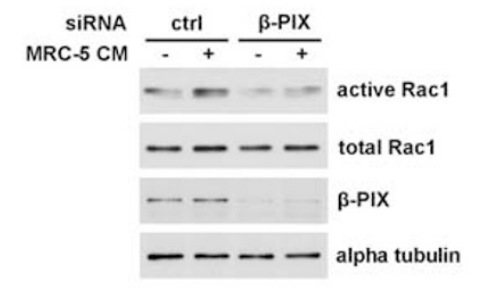

d

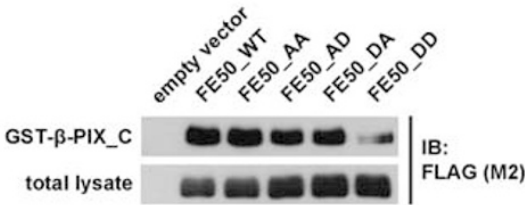

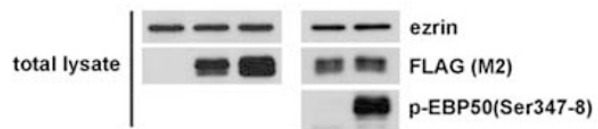

Figure 5 Phosphorylation of EBP50 serine $347 / 348$ reduces EBP50 binding to $\beta$-PIX. (a) HEK293 cells were transiently transfected with the indicated plasmids and cell lystates were immunoprecipitated with mouse anti-FLAG antibody (M2) and then the immunoprecipitates were processed for western blotting analysis using either mouse anti-Myc or the rabbit anti-FLAG antibodies. (b) Rac1 activation assay was performed in MDCK cells transiently expressing control oligonucleotides or siRNA targeted at $\beta$-PIX in the absence or presence of MRC-5 fibroblasts conditioned medium. The data shown here is a representative result from three independent experiments. (c) Cell lysates from MDCK EBP50 knockdown clone (siE50_16) and the siRNA-resistant revertants siE50_16-reAA, siE50_16-reDD and siE50_16-reWT (with or without PMA) were incubated with glutathione beads, which had been loaded with GST, GST-fused C-terminal one-third of $\beta$-PIX deleted off its PDZ-binding motif (GST- $\beta$-PIX_DC) and C-terminal one-third of $\beta$-PIX (GST- $\beta$-PIX_C). The initial cell lysates and the pulled-down material were examined by western blotting analysis using mouse anti-FLAG (M2), ezrin or rabbit pEBP50(Ser347-8) antibodies. (d) HEK293 cells were transiently transfected with the indicated plasmids. After $24 \mathrm{~h}$, the cell lysates were incubated with glutathione beads, which had been loaded with GST-fused C-terminal one-third of $\beta$-PIX. The pulled-down material was examined by western blotting analysis for mouse anti-FLAG antibody (M2)

of both serine 347 and 348 with aspartic acid decreased the interaction between EBP50 and $\beta$-PIX, aspartic acid substitution of either serine 347 or 348 had little effect (Figure $5 d$ ). Furthermore, EBP50 mutants containing an aspartic acid substitution of either serine 347 or 348 did not significantly affect Rac1 activation by MRC5 conditioned medium (Supplementary Figure 5). These results, together with our finding of the effect of double serine phosphorylation on the interaction between EBP50 and ezrin (Figure 3), indicate that EBP50 phosphorylation at serine 347 and 348 potentiates the dissociation of the EBP50-ezrin- $\beta$-PIX complex and reduces Rac1 activation.

Cell detachment from the substratum induces phosphorylation of EBP50 at serine $347 / 348$ and downregulates Rac1 activity by disassembly of the EBP50-ezrin- $\boldsymbol{\beta}$-PIX complex. Because PMA increased phosphorylation of EBP50 (Figures $2 b, 3 b$, and $5 c$ ), we hypothesized that there should be a physiological setting when Rac1 activity is downregulated through EBP50 phosphorylation. To test this hypothesis, we examined EBP50 phosphorylation in MDCK cells grown in suspension culture, a condition that induces anoikis ${ }^{31}$ and inhibits Rac1 activity $^{32}$ (Supplementary Figure 6). Substratum detachment indeed induced a moderate but significant increase of EBP50 phosphorylation, which was even more pronounced in cells treated with calyculin $A$, a potent inhibitor of PP2A and protein phosphastase 1 (PP1) (Figure 6a). Because EBP50 phosphorylation was inhibited by Gö 6976, a classical type PKC inhibitor (Figure 6b), PKC could be the kinase responsible for EBP50 phosphorylation in MDCK cells in suspension culture. As PKC has been shown to be associated with PP2A, and its activity is inhibited through dephosphorylation by PP2A, ${ }^{33}$ the effect of calyculin $A$ on EBP50 phosphorylation might be indirectly through the loss of PP2A inhibitory regulation of PKC or directly through a putative phosphatase acting on EBP50 per se. To distinguish between these possibilities, we examined the level of EBP50 phosphorylation in cells grown in suspension culture that were first treated with Gö 6976 to suppress the PKC function and then treated with calyculin A. The level of EBP50 phosphorylation at serine 347/348 under these combined inhibitor treatment was similar to that when cells were incubated with only calyculin A (Figure 6b). This result indicates that calyculin $A$ inhibits a phosphatase, acting directly on EBP50 but not on PKC. Thus, the moderate increase in the level of EBP50 phosphorylation in cells in suspension culture cells (in the absence of calyculin A) was the net result from the opposing activities of a kinase and a phosphatase (Figure 6a). 


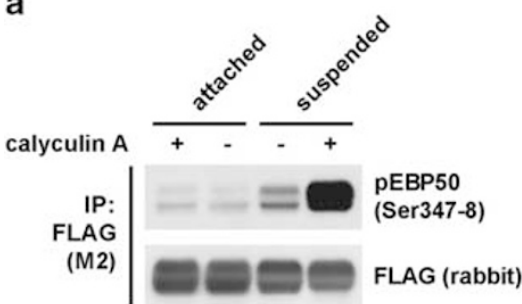

C
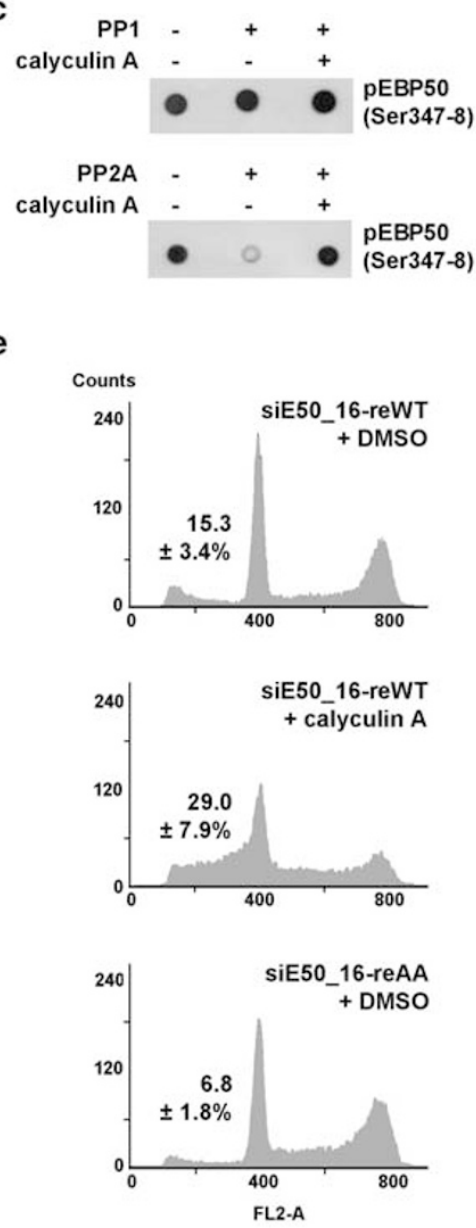

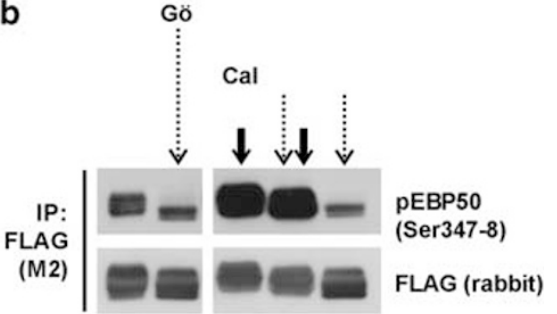

d

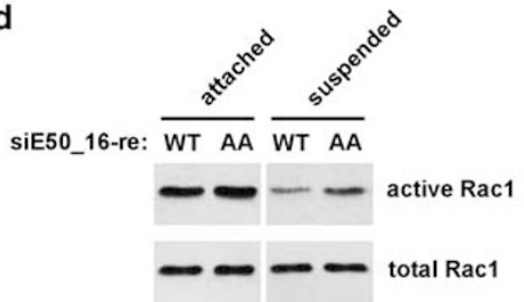

f
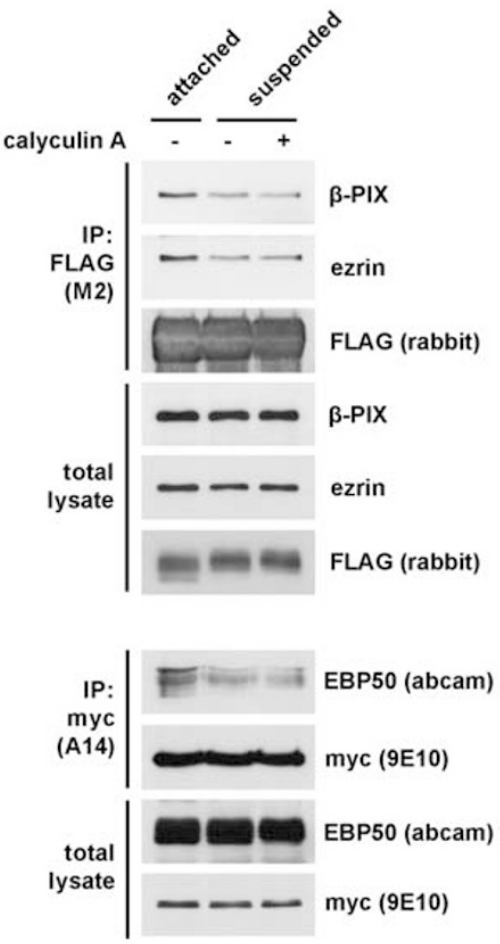

Figure 6 Loss of substratum interaction induces phosphorylation of EBP50 at serine 347/348 position and downregulates Rac1 activity. (a) MDCK cells stably expressing FLAG-tagged EBP50 were either kept attached to the substratum or grown in suspension culture for $2 \mathrm{~h}$. Cells were treated with either the phosphatase inhibitor calyculin $\mathrm{A}$ $(40 \mathrm{nM})$ or the solvent control DMSO for $20 \mathrm{~min}$. Cell lysates were immunoprecipitated with mouse anti-FLAG antibody (M2) and then the immunoprecipitates were processed for western blotting analysis using either rabbit anti-FLAG or EBP50 phospho-specific antibodies. (b) MDCK cells stably expressing FLAG-tagged EBP50 were kept detached from substratum for $130 \mathrm{~min}$ in DMSO (lane 1) or $5 \mu \mathrm{M}$ Gö 6976 (lane 2). Alternatively, the suspension cells were incubated with $40 \mathrm{nM}$ calyculin A for the last $20 \mathrm{~min}$ (lane 3), with $5 \mu \mathrm{M}$ Gö 6976 for the last $40 \mathrm{~min}$ (lane 5), or a combination of both (lane 4) before cell lysates were collected for immunoprecipitation using mouse anti-FLAG (M2) antibody, and processed for western blotting analysis with rabbit anti-FLAG or EBP50 phospho-specific antibodies. (c) The synthetic phosphopeptide (RAHQKR(pS)(pS)KRAPQM) was treated with purified PP1 or PP2A phosphatase in the absence (middle dots) or presence (right dots) of Calyculin A (100 nM), and processed for immuno-dot blot analysis with the EBP50 phospho-specific antibody. MDCK revertants stably expressing siRNA-resistant FE50_WT and FE50_AA constructs were kept attached or detached from substratum for $2 \mathrm{~h}$ before being processed for the Rac1 activity assay (d) or flow cytometry (e) to measure sub-G1 cell population (The data shown were means \pm S.E. of a representative triplicated assay). (f) MDCK stably expressing FE50_WT (upper panel) and Myc-tagged $\beta$-PIX (lower panel) were kept attached or detached from the substratum for $2 \mathrm{~h}$ with or without Calyculin $\mathrm{A}(40 \mathrm{nM})$ for last $20 \mathrm{~min}$ before cell lysates were isolated and immunoprecipitated with mouse antiFLAG antibody (M2) or rabbit anti-Myc antibody (A14); immune complexes were analyzed by western blotting analysis for $\beta$-PIX, ezrin (upper panel) or EBP50 (lower panel), respectively 
When we treated the antigenic peptide phosphorylated on serine $347 / 348$ with purified PP2A, the immunogenicity of the peptide was greatly reduced (Figure $6 \mathrm{c}$ ). The immunogenicity of this peptide was preserved when calyculin $A$ was present in the reaction, indicating that PP2A was responsible, and not other enzymatic activities contaminated in the preparation. In contrast, treatment with purified PP1 did not affect the immunogenicity of the peptide (Figure 6c). Taken together, these results indicate that there exists not only a cell suspension culture-dependent PKC activity towards EBP50 but also a counter-regulatory phosphatase activity in cells deprived of substratum attachment.

The effect of EBP50 phosphorylation at serine 347/348 on Rac1 activity was studied in stable MDCK clones expressing FE50_reWT or FE50_reAA in suspension culture. There was a greater degree of Rac1 activation in FE50_reAA-expressing cells than in FE50_reWT-expressing cells (Figure 6d). Rac1 activation is a known survival factor against anoikis ${ }^{34}$ and, as expected, we found that there was a smaller sub-G1 population in the FE50_reAA-expressing cells (Figure 6e). In line with our hypothesis that enhanced EBP50 phosphorylation would downregulate the pro-survival Rac1 activity, calyculin A treatment almost doubled the number of apoptotic cells (Figure 6e). To further link Rac1 activity to EBP50-dependent anoikis, we took advantage of a previously well-characterized MDCK clone stably expressing constitutively active Rac1 (Rac1V12) by tetracyclinerepressible system. ${ }^{35}$ Inducible Rac1 activation mitigated the enhanced anoikis seen in EBP50 knockdown cells (Supplementary Figure 7). This result demonstrates that diminished Rac1 activity indeed contributes to the anoikis after EBP50 knockdown.

Because EBP50 phosphorylation results in a decreased interaction of EBP50 with ezrin and $\beta$-PIX (Figures 3 and 5), and EBP50 is increasingly phosphorylated at serine $347 / 348$ in MDCK cells in suspension culture, we expected that the interaction between EBP50, $\beta$-PIX and ezrin should undergo a corresponding change in cells grown in suspension culture. Indeed, co-immunoprecipitation of proteins from the cells in suspension culture showed that both $\beta$-PIX and ezrin decreased their association with EBP50 (Figure 6f), and a corresponding increase in EBP50 phosphorylation (data not shown).

To explore whether this phenomenon was found in cells other than MDCK cells, we extended our study to HaCaT cells in which anoikis was first demonstrated. ${ }^{31}$ Although EBP50 phosphorylation at serine $347 / 348$ in cells in suspension culture was not evident, calyculin $A$ treatment revealed increased EBP50 phosphorylation similar to what was observed in MDCK cells (Supplementary Figure 8a). Furthermore, knockdown of EBP50 resulted in increased levels of apoptosis when cells were detached from the substratum (Supplementary Figure 8b). Compatible with our hypothesis that EBP50 phosphorylation disrupts the interaction between EBP50, $\beta$-PIX and ezrin, calyculin A increased the level of anoikis presumably due to a concomitant decrease in Rac1 activity (Supplementary Figure 8c). Thus, EBP50 phosphorylation regulates the level of Rac1 activation and anoikis in $\mathrm{HaCaT}$ cells, similar to the effects in MDCK cells (Figure 6).

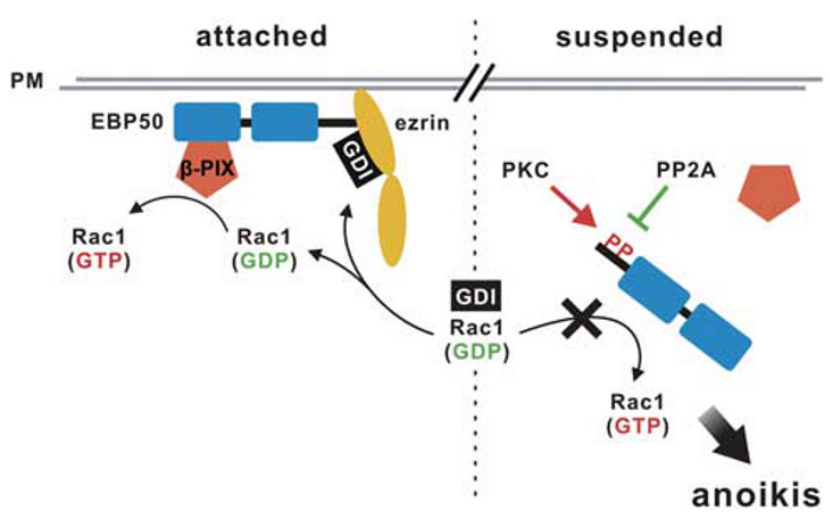

Figure 7 Schematic model depicting how loss of substratum interaction enhances phosphorylation of EBP50 at serine 347/348 and downregulates Rac1 activity to induce anoikis. EBP50 provides a scaffold to facilitate complex formation with $\beta$-PIX and ezrin at the plasma membrane (PM). This complex sequesters GDI from cytosolic GDP-loaded Rac1 through ezrin. The PDZ domain of EBP50 also interacts with $\beta$-PIX, which induces the exchange of GDP with GTP on Rac1, and activated Rac1 generates a survival message. Upon detachment from the substratum, counteracting PKC and PP2A phosphorylates and dephosphorylates, respectively, serine $347 / 348$ in the ezrin-binding region of EBP50 that results in a net increase in the level of EBP50 phosphorylation, subsequently dissociating the EBP50-ezrin- $\beta$-PIX complex, and a failure in Rac1 activation. The loss of Rac1 pro-surviving activity induces anoikis

\section{Discussion}

EBP50 participates in Rac1 activation by releasing the inhibitory effect of RhoGDI, and facilitating GDP to GTP nucleotide exchange through the interaction of EBP50 with ezrin and $\beta$-PIX (Figure 7). ${ }^{18,19}$ Interactions with plasma membrane lipid PIP2 converts ezrin from an auto-inhibitory into an open active conformation, and subsequent PKCmediated phosphorylation of ezrin further stabilizes the activated state of ezrin. ${ }^{36}$ Active ezrin then sequesters RhoGDI to release small GTPases for activation by exchanging GDP for GTP. ${ }^{37}$ We showed in this study that PKC also phosphorylates EBP50 when cells are detached from the substratum, which results in the disassembly of the interaction between EBP50, ezrin and $\beta$-PIX to terminate Rac1 activity. This finding implies that PKC activity is not only a positive regulatory signal for Rac1 activation but it also terminates Rac1 activation by phosphorylating EBP50. This mechanism fulfills the requirement of small GTPases as molecular switches to maintain adequate activation of various intracellular signals. The role of EBP50 as a scaffold protein that both facilitates and restricts a specific signaling is not without precedent. The canonical scaffold protein KSR (kinase suppressor of Ras) tethers Raf, MEK and Erk to facilitate a three-tiered MAPK pathway. After a series of phosphorylation events, activated Erk phosphorylates both KSR and Raf to disrupt their interaction and creates an inhibitory feedback mechanism to shut down the MAPK signaling. ${ }^{1}$

Our data amazingly revealed a cryptic pair of kinase and phosphatase activities on EBP50 after deprivation of cell contact to substratum. The phosphorylation of EBP50 by PKC was largely opposed by an elusive PP2A phosphatase because the extent of EBP50 phosphorylation at canine serine $347 / 348$ in MDCK cells was robustly revealed by calyculin A (Figures 6a and b), a PP1 and PP2A phosphatase 
inhibitor. This indicates that loss of substratum attachment elicits an increase in both PKC and PP2A phosphatase activities. The net effect is that EBP50 represses Rac1 survival signaling. It has been proposed that PP2A-type phosphatase associates with conventional PKC and suppresses its enzymatic activity by dephosphorylating the critical phosphoserine residue in the hydrophobic C-terminal. ${ }^{33}$ It is therefore possible that the augmented EBP50 phosphorylation we observed in suspension cultured cells after calyculin A treatment was an indirect effect of an inhibitory phosphatase on upstream PKC rather than inhibition of a phosphatase that was acting directly on EBP50. Our results favor a direct involvement of phosphatase in EBP50 dephosphorylation because Gö 6976, a classical type PKC inhibitor, did not decrease EBP50 phosphorylation after calyculin A treatment (Figure 6b) and the antigenic phosphopeptide lost its immunogenicity after addition of purified PP2A (Figure 6c). It will be interesting to examine whether any PP2A phosphatases contain a PDZ-binding motif as a potential EBP50-binding candidate, which could help to identify the phosphatase involved in dephosphorylating these critical serines in EBP50.

EBP50 is phosphorylated by GRK6A and PKC. ${ }^{14}$ Interestingly, these two kinases are either activated by downstream effector of GPCR or by critical regulators of GPCR signaling. ${ }^{38}$ Several GPCRs bind to EBP50 through their C-terminal PDZbinding motif. $^{13}$ Upon ligand binding, GPCRs undergo a conformational change, which activates protein kinase that phosphorylates GPCR. It will be interesting to test how EBP50 phosphorylation by PKC or other kinases affects the regulation of internalization, downregulation through targeting to lysosomal degradation, and recycling of the GPCRs. EBP50 has been shown to stabilize GPCRs, including $\beta 2$-adrenergic, $\mathrm{PTH}$ and $\kappa$-opioid receptors, at the membrane by tethering these proteins to membrane cytoskeleton via ezrin, and therefore reducing receptor internalization. ${ }^{8,9,39}$ This conclusion was based on an EBP50 mutant deleted of its ezrinbinding region, which had also lost membrane-tethering capacity. However, we were unable to identify an EBP50deletion mutant that had this type of regulation. Instead, our finding that phosphorylation at the ezrin-binding region of EBP50 diminishes binding between EBP50 and ezrin (Figure 3), and induces a cytosolic redistribution of EBP50 (Figures 1 and 2), provides a new mechanistic insight into the role of EBP50 as a regulator of GPCR internalization.

We previously demonstrated that the canonical apicalmarker protein gp135/podocalyxin, a type I transmembrane protein, interacts with EBP50 early in the biosynthetic pathway to facilitate its apical sorting and targeting in polarized MDCK cells. ${ }^{40}$ Furthermore, an earlier report indicates that surface delivery of the transient-receptor-potential canonical protein TRPC4, a calcium channel with six transmembrane segments, also depends on its interaction with EBP50. ${ }^{27}$ It will be interesting to learn whether EBP50 phosphorylation affects the trafficking of its interacting proteins early in the biosynthetic pathway.

\section{Materials and Methods}

Plasmid construction. FLAG-tagged canine EBP50 and a series of EBP50 mutant constructs were derived from pCMV-Tag2A (Agilent Technologies, Palo
Alto, CA, USA). Myc-tagged human $\beta$-PIX was derived from pcDNA3.1 $(+)$ (Invitrogen, Carlsbad, CA, USA). GST-tagged constructs pGEX2T- $\beta$-PIX_C and pGEX2T- $\beta$-PIX_DC were described previously. ${ }^{19}$ All of these constructs were verified by automatic DNA nucleotide sequencing and the detailed sequencing information is available (http://homepage.ntu.edu.tw/ d95448004/2011_Chen/ default.htm)

EBP50 knockdown by siRNA approach. Expression levels of canine EBP50 and $\beta$-PIX were knocked down using synthetic RNA duplexes (Stealth siRNA for EBP50 (5'-GAGACUGAUGAGUUCUUCAAGAAAU-3'), and $\beta$-PIX $\left(5^{\prime}\right.$-GCUAUUACAAUGUGGUGCUACAGAA-3')) and Lipofectamine 2000 as the transfection vehicle, according to the manufacturer's instruction (Invitrogen). Stable EBP50 knockdown cell lines were generated by transfecting pSilencer 1.0-derived plasmids (Ambion, Austin, TX, USA) expressing siRNA, targeting against $5^{\prime}$-GGAGACTGATGAGTTCTTC- $3^{\prime}$ and 5'-ATGAACTCTTCAGCAACCT- $3^{\prime}$, respectively. Subsequently, stable cell lines were selected and maintained in $800 \mu \mathrm{g} / \mathrm{ml}$ G418 (Sigma-Aldrich, St. Louis, MO, USA).

Antibodies and reagents. PMA was from Promega (Madison, WI, USA). Gö 6976 was from Merck KGaA (Darmstadt, Germany). Protease inhibitor cocktail was from Sigma-Aldrich. Calyculin A was from Cell Signaling Technology (Beverly, MA, USA). Purified PP1 and PP2A were from Millipore (Billerica, MA, USA). Hybridoma cells secreting anti-gp135 (3F2/D8) and tight junction marker ZO-1 (R40.76) monoclonal antibodies were gifts from Dr. George Ojakian (SUNY Downstate Medical Center, New York, NY, USA) and Dr. Dan Goodenough (Harvard Medical School, Boston, MA, USA), respectively. Anti-Myc hybridoma cells (9E10) were from ATCC (Manassas, VA, USA). Rabbit anti-FLAG polyclonal and mouse anti-alpha tubulin monoclonal antibodies were gifts from Dr. Sheng-Chung Lee (National Taiwan University, Taipei, Taiwan). Mouse anti-Rac1 antibody was from Millipore. Mouse anti-FLAG monoclonal antibody (M2) was from Sigma-Aldrich. Rabbit anti- $\beta$-PIX polyclonal antibodies were from Cell Signaling Technology. Rabbit anti-Myc polyclonal antibodies (A14) were from Santa Cruz Biotechnology (Santa Cruz, CA, USA). Rabbit anti-EBP50 polyclonal and mouse anti-ezrin monoclonal antibodies were from Abcam (Cambridge, MA, USA) and Lab Vision (Fremont, CA, USA), respectively. Anti-phospho-EBP50 (Ser347 and 348) polyclonal antibodies were generated by immunizing rabbits with a synthetic phosphopeptide (RAHQKR(pS)(pS)KRAPQM) and were further purified by affinity chromatography.

Cell culture and transfection. MDCK type II, HEK293 cells and human embryonic lung fibroblast (MRC-5) cells were cultured in Dulbecco's modified Eagles medium (DMEM) supplemented with $10 \%$ fetal calf serum at $37^{\circ} \mathrm{C}$ in a humidified incubator containing $5 \% \mathrm{CO}_{2}$. Transfection was performed using Lipofectamine 2000 (Invitrogen) according to the manufacturer's instruction.

Immunofluorescence and image acquisition. Confluent monolayers of MDCK cells were grown on Transwell filters (Corning, Corning, NY, USA) in DMEM/ FCS. Cells were washed twice with PBS $\left(0.9 \mathrm{mM} \mathrm{CaCl}_{2}, 2.7 \mathrm{mM} \mathrm{KCl}, 1.5 \mathrm{mM}\right.$ $\mathrm{KH}_{2} \mathrm{PO}_{4}, 0.5 \mathrm{mM} \mathrm{MgCl}_{2}, 137 \mathrm{mM} \mathrm{NaCl}$ and $8.1 \mathrm{mM} \mathrm{Na}_{2} \mathrm{HPO}_{4}$ ) and then fixed for 20 min at room temperature (RT) in $3.7 \%$ paraformaldehyde in PBS. Cells were then washed twice in PBS at RT for 10 min and extracted with CSK buffer $(50 \mathrm{mM} \mathrm{NaCl}$, $300 \mathrm{mM}$ sucrose, $10 \mathrm{mM}$ Pipes, $\mathrm{pH} 6.8,3 \mathrm{mM} \mathrm{MgCl}_{2}$ and $0.5 \%$ (vol/vol) Triton X-100) at RT for $10 \mathrm{~min}$. Cells were washed twice in PBS at RT for $10 \mathrm{~min}$. Cells were blocked in PBS containing $1 \% \mathrm{BSA}, 10 \%$ goat serum and $50 \mathrm{mM} \mathrm{NH}_{4} \mathrm{Cl}$ for $1 \mathrm{~h}$ at RT. After blocking, cells were washed briefly in PBS and $0.2 \%$ BSA and then incubated in primary antibody solution for $1 \mathrm{~h}$ at RT. Cells were then washed three times in PBS and $0.2 \% \mathrm{BSA}$ and incubated in FITC- or rhodamine-conjugated goat secondary antibody solution for $1 \mathrm{~h}$ at RT. Cells were washed three times in PBS and $0.2 \% \mathrm{BSA}$ and mounted in Vectashield (Vector Laboratories, Burlingame, CA, USA). X-Z section images were acquired with Zeiss LSM510 confocal microscope (Carl Zeiss Jena Gmbh, Jena, Germany) using a $63 \times 1.25$ NA oil immersion objective.

Immunoprecipitation. Cells were lysed and extracted at $4{ }^{\circ} \mathrm{C}$ for $30 \mathrm{~min}$ in RIPA or CSK buffer containing protease inhibitor (Sigma-Aldrich) and phosphatase inhibitor (Merck KGaA). Subsequently, an aliquot of cell lysates was incubated with rabbit anti-Myc (A14) antibody-conjugated NHS-activated Sepharose (General Electric, Fairfield, CT, USA) or mouse anti-FLAG M2 affinity resin at $4{ }^{\circ} \mathrm{C}$ for $4 \mathrm{~h}$. Beads were then collected and washed three times with the same extraction buffer and eluted by boiling in SDS sample buffer. Protein elutes were separated by SDS-PAGE and processed for western blotting analysis. 
Western blotting analysis. Protein samples were denatured in SDS sample buffer and separated by SDS-PAGE. Following electrophoresis, proteins in the gel were transferred onto nitrocellulose membrane (PerkinElmer, Norwalk, CT, USA) at $400 \mathrm{~mA}$ for $3 \mathrm{~h}$. The membrane was blocked in $5 \%$ non-fat milk/TBST (Tris-buffered saline plus $0.05 \%$ Tween-20) for $1 \mathrm{~h}$ at RT and incubated with specific primary antibody diluted in $1 \%$ non-fat milk/TBST at RT for $1 \mathrm{~h}$ or overnight at $4{ }^{\circ} \mathrm{C}$. The membrane was washed three times with TBST, $10 \mathrm{~min}$ each, and then incubated with HRP-conjugated secondary antibody (General Electric; Millipore) for $1 \mathrm{~h}$ at RT. After three washes, $10 \mathrm{~min}$ each, the membrane was developed using ECL reagents (PerkinElmer) and exposed onto X-ray films (General Electric).

Subcellular fractionation. Cells were washed with Tris-buffered Saline once and incubated for $10 \mathrm{~min}$ with ice-cold hypotonic buffer (10 mM HEPES at pH $7.3,5 \mathrm{mM} \mathrm{KCl}, 1.5 \mathrm{mM} \mathrm{MgCl}_{2}, 1 \mathrm{mM}$ DTT and protease inhibitors). Following, cells were scraped and homogenized on ice with Dounce homogenizer (20 strokes with a tight pestle). To separate the membrane and cytosolic fractions, homogenate was centrifuged at $700 \mathrm{~g}$ for $3 \mathrm{~min}$ at $4^{\circ} \mathrm{C}$. The supernatant was further centrifuged at $16000 \mathrm{~g}$ for $30 \mathrm{~min}$ at $4^{\circ} \mathrm{C}$ to generate a cytosolic fraction (supernatant) and membranes (pellet). Membranes were gently washed once with ice-cold hypotonic buffer and dissolved in SDS-PAGE sample buffer. ${ }^{41}$ For western blotting analysis, $2 \%$ of the cytosolic fraction and $15 \%$ of the membrane fraction were analyzed.

Rac1 activation assay. The p21-binding domain (PBD) of p21-activated kinase 1 was fused with GST to make a recombinant protein (GST-PBD). The procedure of preparing GST-PBD, processing cellular lysates to perform the pulldown assay, and using anti-Rac1 antibody for western blotting analysis was essentially as described before. ${ }^{19}$ To induce Rac1 activation, cells were serumstarved overnight followed by the treatment of MRC-5 conditioned medium (1:1 dilution in medium) for $10 \mathrm{~min}$.

GST pull-down assay. Recombinant GST- $\beta$-PIX_C and GST- $\beta$-PIX_DC proteins were expressed in Escherichia coli strain BL21 (DE3) pLys and purified on glutathione beads (General Electric) according to standard protocols. To perform pull-down assay, the beads bearing purified GST fusion proteins were mixed with cell lysates and incubated at $4{ }^{\circ} \mathrm{C}$ for $4 \mathrm{~h}$. After washing steps, proteins bound to beads are resolved by SDS-PAGE and analyzed by western blotting.

Cell scattering assay. Cells were seeded on rat tail collagen-coated plates and cultured for $48 \mathrm{~h}$ to allow the formation of single colonies. To stimulate cell scattering, cells were starved in DMEM without serum for $7 \mathrm{~h}$, followed by $16 \mathrm{~h}$ of MRC- 5 conditioned medium treatment in the absence of serum. Phase contrast images were obtained using Leica-DMIL inverted microscope (Leica Microsystems $A G$, Wetzlar, Germany) at $\times 10$.

In vitro dephosphorylation assay. $0.1 \mu \mathrm{g}$ synthetic EBP50 phosphopeptide (RAHQKR(pS)(pS)KRAPQM) was incubated with 0.1 unit of purified PP1 in PP1 reaction buffer (50 mM Tris- $\mathrm{HCl}, \mathrm{pH} 7.0,0.1 \mathrm{mM}$ EDTA, $5 \mathrm{mM}$ DTT, $0.01 \%$ TX-100 and $1 \mathrm{mM} \mathrm{MnCl})^{42}$ or with 0.1 unit of purified PP2A in PP2A reaction buffer (20 mM HEPES pH7.0, $1 \mathrm{mM}$ DTT, $1 \mathrm{mM} \mathrm{MgCl}_{2}$ and $\left.100 \mathrm{mg} / \mathrm{ml} \mathrm{BSA}\right)^{43}$ at $30^{\circ} \mathrm{C}$ for $1 \mathrm{~h}$ in the absence or presence of calyculin $A(100 \mathrm{nM})$. Subsequently, the phosphorylation status of EBP50 peptide was assessed by immuno-dot blot assay using pEBP50(Ser347-8) antibody.

Anoikis assay. MDCK cells were suspended in serum-free DMEM over $2 \%$ agarose-coated petri dishes. After $4 \mathrm{~h}$, cells were collected for propidium-iodide staining and assessed by FACSCalibur flow cytometer (BD Biosciences, San Jose, CA, USA) according to the standard protocols for apoptosis analysis. Anoikic cells were quantified by calculating the sub-G1 population.

\section{Conflict of Interest}

The authors declare no conflict of interest.

Acknowledgements. This study is supported by National Science Council Grants (NSC 97-2320-B-002-048-MY3, and 99-3112-B-002-020) and National Taiwan University Hospital Grants (aNTUH-99P21-1) to T. S. Jou. We thank Professor W James Nelson (Stanford University) for English editing of this manuscript.
1. Good MC, Zalatan JG, Lim WA. Scaffold proteins: hubs for controlling the flow of cellular information. Science 2011; 332: 680-686.

2. Yun $\mathrm{CH}, \mathrm{Oh} \mathrm{S}$, Zizak M, Steplock D, Tsao S, Tse CM et al. cAMP-mediated inhibition of the epithelial brush border $\mathrm{Na}+\mathrm{H}+$ exchanger, $\mathrm{NHE} 3$, requires an associated regulatory protein. Proc Natl Acad Sci USA 1997; 94: 3010-3015.

3. Reczek D, Berryman M, Bretscher A. Identification of EBP50: A PDZ-containing phosphoprotein that associates with members of the ezrin-radixin-moesin family. J Cell Biol 1997; 139: 169-179.

4. Gisler SM, Stagljar I, Traebert M, Bacic D, Biber J, Murer H. Interaction of the type Illa Na/Pi cotransporter with PDZ proteins. J Biol Chem 2001; 276: 9206-9213.

5. Weinman EJ, Wang Y, Wang F, Greer C, Steplock D, Shenolikar S. A C-terminal PDZ motif in NHE3 binds NHERF-1 and enhances CAMP inhibition of sodium-hydrogen exchange. Biochemistry 2003; 42: 12662-12668.

6. Short DB, Trotter KW, Reczek D, Kreda SM, Bretscher A, Boucher RC et al. An apical PDZ protein anchors the cystic fibrosis transmembrane conductance regulator to the cytoskeleton. J Biol Chem 1998; 273: 19797-19801.

7. Hall RA, Premont RT, Chow CW, Blitzer JT, Pitcher JA, Claing A et al. The beta2adrenergic receptor interacts with the $\mathrm{Na}+/ \mathrm{H}+$-exchanger regulatory factor to control $\mathrm{Na}+/ \mathrm{H}+$ exchange. Nature 1998; 392: 626-630.

8. Li JG, Chen C, Liu-Chen LY. Ezrin-radixin-moesin-binding phosphoprotein-50/Na+/H+ exchanger regulatory factor (EBP50/NHERF) blocks U50,488H-induced down-regulation of the human kappa opioid receptor by enhancing its recycling rate. J Biol Chem 2002; 277 : 27545-27552.

9. Wheeler D, Sneddon WB, Wang B, Friedman PA, Romero G. NHERF-1 and the cytoskeleton regulate the traffic and membrane dynamics of $G$ protein-coupled receptors. $J$ Biol Chem 2007; 282: 25076-25087.

10. Takahashi Y, Morales FC, Kreimann EL, Georgescu MM. PTEN tumor suppressor associates with NHERF proteins to attenuate PDGF receptor signaling. EMBO J 2006; 25: 910-920.

11. Lazar CS, Cresson $\mathrm{CM}$, Lauffenburger DA, Gill GN. The $\mathrm{Na}+/ \mathrm{H}+$ exchanger regulatory factor stabilizes epidermal growth factor receptors at the cell surface. Mol Biol Cell 2004; 15: $5470-5480$

12. Shenolikar S, Weinman EJ. NHERF: targeting and trafficking membrane proteins. Am J Physiol Renal Physiol 2001; 280: F389-F395.

13. Georgescu MM, Morales FC, Molina JR, Hayashi Y. Roles of NHERF1/EBP50 in cancer. Curr Mol Med 2008; 8: 459-468.

14. Garbett D, LaLonde DP, Bretscher A. The scaffolding protein EBP50 regulates microvillar assembly in a phosphorylation-dependent manner. J Cell Biol 2010; 191: 397-413.

15. Ueda T, Kikuchi A, Ohga N, Yamamoto J, Takai Y. Purification and characterization from bovine brain cytosol of a novel regulatory protein inhibiting the dissociation of GDP from and the subsequent binding of GTP to rhoB p20, a ras p21-like GTP-binding protein. J Biol Chem 1990; 265: 9373-9380.

16. DerMardirossian C, Bokoch GM. GDIs: central regulatory molecules in Rho GTPase activation. Trends Cell Biol 2005; 15: 356-363.

17. Rossman KL, Der CJ, Sondek J. GEF means go: turning on RHO GTPases with guanine nucleotide-exchange factors. Nat Rev Mol Cell Biol 2005; 6: 167-180.

18. Schmieder S, Nagai M, Orlando RA, Takeda T, Farquhar MG. Podocalyxin activates RhoA and induces actin reorganization through NHERF1 and Ezrin in MDCK cells. J Am Soc Nephrol 2004; 15: 2289-2298.

19. Hsu YH, Lin WL, Hou YT, Pu YS, Shun CT, Chen CL et al. Podocalyxin EBP50 ezrin molecular complex enhances the metastatic potential of renal cell carcinoma through recruiting Rac1 guanine nucleotide exchange factor ARHGEF7. Am J Pathol 2010; 176: 3050-3061.

20. Li J, Poulikakos PI, Dai Z, Testa JR, Callaway DJ, Bu Z. Protein kinase C phosphorylation disrupts $\mathrm{Na}+/ \mathrm{H}+$ exchanger regulatory factor 1 autoinhibition and promotes cystic fibrosis transmembrane conductance regulator macromolecular assembly. J Biol Chem 2007; 282: 27086-27099.

21. Hall RA, Spurney RF, Premont RT, Rahman N, Blitzer JT, Pitcher JA et al. G proteincoupled receptor kinase $6 \mathrm{~A}$ phosphorylates the $\mathrm{Na}(+) / \mathrm{H}(+)$ exchanger regulatory factor via a PDZ domain-mediated interaction. J Biol Chem 1999; 274: 24328-24334.

22. Lau AG, Hall RA. Oligomerization of NHERF-1 and NHERF-2 PDZ domains: differential regulation by association with receptor carboxyl-termini and by phosphorylation. Biochemistry 2001; 40: 8572-8580.

23. He J, Lau AG, Yaffe MB, Hall RA. Phosphorylation and cell cycle-dependent regulation of $\mathrm{Na}+\mathrm{H}+$ exchanger regulatory factor-1 by Cdc2 kinase. J Biol Chem 2001; 276: 41559-41565.

24. Fouassier L, Nichols MT, Gidey E, McWilliams RR, Robin H, Finnigan $\mathrm{C}$ et al. Protein kinase $C$ regulates the phosphorylation and oligomerization of ERM binding phosphoprotein 50. Exp Cell Res 2005; 306: 264-273.

25. Raghuram V, Hormuth H, Foskett JK. A kinase-regulated mechanism controls CFTR channel gating by disrupting bivalent PDZ domain interactions. Proc Natl Acad Sci USA 2003; 100: 9620-9625.

26. Saotome I, Curto M, McClatchey Al. Ezrin is essential for epithelial organization and villus morphogenesis in the developing intestine. Dev Cell 2004; 6: 855-864.

27. Mery L, Strauss B, Dufour JF, Krause KH, Hoth M. The PDZ-interacting domain of TRPC4 controls its localization and surface expression in HEK293 cells. J Cell Sci 2002; 115 (Part 17): 3497-3508. 
28. Masuda M, Kikuchi S, Maruyama T, Sakurai-Yageta M, Williams YN, Ghosh HP et al. Tumor suppressor in lung cancer (TSLC)1 suppresses epithelial cell scattering and tubulogenesis. J Biol Chem 2005; 280: 42164-42171.

29. Ridley AJ, Comoglio PM, Hall A. Regulation of scatter factor/hepatocyte growth factor responses by Ras, Rac, and Rho in MDCK cells. Mol Cell Biol 1995; 15 $1110-1122$.

30. ten Klooster JP, Jaffer ZM, Chernoff J, Hordijk PL. Targeting and activation of Rac1 are mediated by the exchange factor beta-Pix. J Cell Biol 2006; 172: 759-769.

31. Frisch SM, Francis H. Disruption of epithelial cell-matrix interactions induces apoptosis. J Cell Biol 1994; 124: 619-626.

32. Zugasti O, Rul W, Roux P, Peyssonnaux C, Eychene A, Franke TF et al. Raf-MEK-Erk cascade in anoikis is controlled by Rac1 and Cdc42 via Akt. Mol Cell Biol 2001; 21: 6706-6717.

33. Obara K, Mitate A, Nozawa K, Watanabe M, Ito Y, Nakayama K. Interactive role of protein phosphatase $2 \mathrm{~A}$ and protein kinase Calpha in the stretch-induced triphosphorylation of myosin light chain in canine cerebral artery. J Vasc Res 2010; 47: 115-127.

34. Cheng TL, Symons M, Jou TS. Regulation of anoikis by Cdc42 and Rac1. Exp Cell Res 2004; 295: 497-511.

35. Jou TS, Nelson WJ. Effects of regulated expression of mutant RhoA and Rac1 small GTPases on the development of epithelial (MDCK) cell polarity. J Cell Biol 1998; 142 85-100.
36. Fievet BT, Gautreau A, Roy C, Del Maestro L, Mangeat P, Louvard D et al. Phosphoinositide binding and phosphorylation act sequentially in the activation mechanism of ezrin. J Cell Biol 2004; 164: 653-659.

37. Takahashi K, Sasaki T, Mammoto A, Takaishi K, Kameyama T, Tsukita S et al. Direct interaction of the Rho GDP dissociation inhibitor with ezrin/radixin/moesin initiates the activation of the Rho small G protein. J Biol Chem 1997; 272: 23371-23375.

38. Delom F, Fessart D. Role of phosphorylation in the control of clathrin-mediated internalization of GPCR. Int J Cell Biol 2011; 2011: 246954.

39. Cao TT, Deacon HW, Reczek D, Bretscher A, von Zastrow M. A kinase-regulated PDZdomain interaction controls endocytic sorting of the beta2-adrenergic receptor. Nature 1999; 401: 286-290.

40. Yu CY, Chen JY, Lin YY, Shen KF, Lin WL, Chien CL et al. A bipartite signal regulates the faithful delivery of apical domain marker podocalyxin/Gp135. Mol Biol Cell 2007; 18: 1710-1722.

41. Boulter E, Garcia-Mata R, Guilluy C, Dubash A, Rossi G, Brennwald PJ et al. Regulation of Rho GTPase crosstalk, degradation and activity by RhoGDI1. Nat Cell Biol 2010; 12: 477-483.

42. Li L, Kozlowski K, Wegner B, Rashid T, Yeung T, Holmes C et al. Phosphorylation of TIMAP by glycogen synthase kinase-3beta activates its associated protein phosphatase 1. J Biol Chem 2007; 282: 25960-25969.

43. Lee T, Kim SJ, Sumpio BE. Role of PP2A in the regulation of p38 MAPK activation in bovine aortic endothelial cells exposed to cyclic strain. J Cell Physiol 2003; 194: 349-355.

Supplementary Information accompanies the paper on Cell Death and Differentiation website (http://www.nature.com/cdd) 